\title{
The spirit ofDakar: a call for action on malaria
}

Sir - Malaria has been a problem for too long. More than a million people still die from it every year, most of them young children. Nine out of ten of these deaths occur in Africa, where resistance to chloroquine is spreading, diminishing the impact of a drug which in the past has helped to limit the damage wreaked by the disease. Resistance is also increasing to Fansidar, the only other drug affordable for widespread use in Africa. This grim situation prompted an unprecedented meeting earlier this year in Dakar, Senegal, where, for the first time, scientists from both French- and Englishspeaking parts of Africa and scientists from the North came together to confront malaria.

The challenge is enormous. New approaches are needed to control malaria and its mosquito vectors. New drugs must be found to prevent and treat the disease, and vaccines need to be developed. This requires research at every level; these efforts benefit from cooperation. One example of an international cooperative effort is the sequencing of the Plasmodium falciparum genome which will accelerate drug and vaccine discovery and may encourage bright young minds into the field.

The urgent need is to put malaria on the scientific, media and political agenda, and in particular to identify it as a priority for research, both in the developed North and in those areas of the South where the disease is endemic. At the same time, the resources to pursue these goals will remain restricted.

Research is critical for the development of tools for disease control and for their effective deployment. In smallpox, for example, even with the vaccine, the critical insight that it was necessary to vaccinate people only in the vicinity of cases, not the entire population, resulted in cost-effective deployment of staff and resources. In malaria, where the biology of the adversary is more complex, and the existing tools less effective, the interplay between research and control strategies is even more crucial.

The Dakar meeting endorsed the principle that sustained research on malaria in Africa should be a key component of any strategy for tackling the disease. More opportunities for training African scientists as well as for strengthening the research infrastructure will be crucial for building collaborations between African scientists and their partners in the North. Trained African scientists will also be critical for future field trials and interventions.

One unprecedented opportunity to boost research in Africa at a relatively modest cost is offered by the Internet. An immediate challenge is to identify the systems and resources needed to provide research centres in Africa with access to e-mail and other Internet services. This would allow African scientists to access the scientific information they need, and to communicate quickly and cheaply with colleagues elsewhere.

Electronic networks are an essential aid in building collaborative research networks, for which the need is absolutely compelling if we are to tackle malaria - a disease

characterized by highly variable patterns of transmission, pathology and drug resistance in different parts of Africa.

It is only by creating joint repositories of materials - such as strains, reagents and insects - and by common protocols that observations made at various locations and laboratories can be compared. Multi-centre clinical research is also needed to develop or improve therapies for complications of malaria, such as anaemia and cerebral malaria.

Recognizing these needs, the Dakar meeting proposed that sponsoring agencies should promote collaborative research efforts both among African scientists and between scientists in the North and South. An announcement has been made about letters of interest ${ }^{1}$ for collaborative research projects. These will be reviewed in July.

A few examples of priorities ${ }^{2}$ for longterm research are provided here.

- Vaccines could be the most cost-effective way to control malaria, but we need both research in the North and collaboration with African scientists to develop candidate vaccines which can be tested in centres in Africa. Studies on epidemiology, parasite polymorphism and mechanisms of immunity in Africa are also a prerequisite for any trials.

- Antimalarial drugs must be developed to replace those becoming obsolete. This will require incentives to attract participation by the pharmaceutical industry and novel approaches to drug development, including production and testing of drugs in Africa. - The recent development of pyrethrumimpregnated bed nets holds great promise for controlling malaria, but the social and economic factors for their introduction need to be studied in detail. And bed nets cannot be simply introduced, but need to be accompanied by long-term studies to assess their impact on mortality and mosquito resistance to pyrethrum.

- Similarly, case management — recognizing that a sick child has malaria and providing effective treatment in the home - requires contributions from both the social and biological sciences.

The coming months offer what seems to be a window of opportunity for boosting international cooperation in malaria research as a component of overall control. The African regional office of the World Health Organization with support from the World Bank is considering the conclusions reached at Dakar as part of the development of a long-term, African-developed and African-led malaria control strategy. The Organization of African Unity (OAU) will hold its annual meeting in June, and malaria will be on the agenda. The same month, the G-7 countries will discuss emerging and reemerging infectious diseases, including malaria.

In the spirit of the Dakar meeting, we recognize that control of malaria in Africa will require a long-term collaboration between scientists in the North and South and the involvement of many more countries than the initial group which took part in the Dakar meeting. It will require commitments from the industrialized countries to funding, and from African leaders to support scientists and health and research infrastructure in their countries.

The fact that the Dakar meeting was opened by the President of Senegal and closed by its Minister of Health seems to symbolize the growing awareness by leaders in Africa of the need to support research efforts by both indigenous scientists and their colleagues abroad. The international community has a responsibility to recognize malaria, like the AIDS pandemic, as a major challenge and to commit itself to do more to prevent the disaster that looms over Africa. Jean-Marie Bruno (French Ministry of Cooperation); Richard Feachem (World Bank); Tore Godal (TDR, WHO); Thomas Nchinda (elected representative of African scientists for post-Dakar efforts); Bridget Ogilvie (Wellcome Trust); Barend Mons (Netherlands Organization for Scientific Research); Robert Mshana (Organization of African Unity); George Radda (Medical Research Council, UK); Ebrahim Samba (WHO, Brazzaville); Maxime Schwartz (Institut Pasteur); Harold Varmus (National Institutes of Health, USA); The Dakar Scientific Advisory Committee: Samba Diallo (Senegal), Ogobara Doumbo (Mali), Brian Greenwood (United Kingdom), Wenceslaus Kilama (Tanzania), Louis H. Miller (USA), Luiz Pereira da Silva (France)

Correspondence for the group should be addressed to: Louis $\mathrm{H}$. Miller, Bld. 4, Room 126, NIAID, NIH, Bethesda, Maryland 20892, USA; fax (301) 402-0079; e-mail: Louis_Miller@nih.gov

\footnotetext{
Request for letters of interest:

http://www.niaid.nih.gov/dmid/mal_Itr_en.htm

Priorities from the Dakar meeting can be obtained at:
}

http://www.niaid.nih.gov/dmid/malafr/ 